\title{
Malnutrition as an important risk factor for drug-induced liver injury in patients on anti-tubercular therapy: an experience from a tertiary care center in South India
}

\author{
Nihal Ali ${ }^{1}$, Nitin Gupta ${ }^{2,3}$, Kavitha Saravu ${ }^{2,3, *}$ \\ ${ }^{1}$ Department of Medicine, Kasturba Medical College, Manipal, Manipal Academy of Higher Education, Karnataka, India; \\ ${ }^{2}$ Department of Infectious diseases, Kasturba Medical College, Manipal, Manipal Academy of Higher Education, Karnataka, India; \\ ${ }^{3}$ Manipal Center for Infectious Diseases, Prasanna School of Public Health, Manipal Academy of Higher Education, Manipal, Karnataka, India.
}

SUMMARY Drug-induced liver injury (DILI) due to anti-tubercular treatment (ATT) leads to increased morbidity and mortality in patients with tuberculosis (TB). The aim of this study was to find the impact of malnutrition on the development of DILI. This was a prospective cohort study (September 2017 to August 2019) in which all newly diagnosed in-patients with tuberculosis above the age of 18 years were included. Those patients with a body mass index (BMI) of $<18.5 \mathrm{~kg} / \mathrm{m}^{2}$ were considered malnourished. The patients were monitored for the development of DILI. Liver function tests were done at the baseline (before initiation of ATT), on the third day and at discharge in all the patients. Chi-square tests and conditional multiple logistic analysis was performed to identify risk factors associated with DILI. Out of the 319 subjects who were enrolled, a total of 138 patents chose to follow up at our hospital. A total of 14 patients $(10 \%)$ developed DILI. The median time to onset of DILI was found to be ten days. Extra-pulmonary TB, low BMI and high baseline liver enzyme levels had a significant association with the development of DILI $(p<0.05)$. Low serum albumin had increased odds ratio but wasn't statistically significant. Malnutrition is an important risk factor for TB-DILI.

Keywords aspartate transaminase, alanine transaminase, body mass index, tuberculosis, adverse drug reaction, nutrition

\section{Introduction}

Tuberculosis (TB) is an epidemic of massive proportions and is a significant cause of concern in a developing country like India. With an incidence of approximately 2.8 million cases in 2018 , India accounts for almost a quarter of the world's TB affected population (1). Malnutrition in TB is both a cause and an effect. Malnutrition leading to dysfunction of the immune system is one of the most frequent causes of secondary immunodeficiency worldwide (2). It must be recognized as one of the driving reasons for the slower decline of the TB epidemic in India despite all the steps taken nationally towards TB eradication (3). Given the multifaceted nature of this two conditions, tuberculosis and malnutrition, it is of great importance to understand the link between the two and ensure that high-quality treatment can be provided to these patients with minimal interruptions. Drug-induced liver injury (DILI) is a significant cause of treatment interruption in people on anti-tubercular treatment (ATT) (4). This leads to increased morbidity and mortality among affected patients. It is hence imperative to consider the implications that coincident malnutrition may have on the impact of DILI in patients with tuberculosis, especially considering that the incidence of DILI is far more in developing countries where malnutrition is rampant (5). The study aimed therefore, to find the impact of malnutrition on the development of DILI.

\section{Materials and Methods}

This was a cohort study conducted between September 2017 and August 2019 at a tertiary care hospital in South India after taking approval from the Institute's Ethics committee (approval number: 660/2017). All newly diagnosed in-patients with tuberculosis (pulmonary and extra-pulmonary) above the age of 18 years were included in the study. Those patients with a past history of tuberculosis were excluded. A brief clinical history, 
examination and baseline laboratory parameters of all the recruited patients were obtained and recorded according to a pre-defined questionnaire. Those patients with a body mass index (BMI) of $<18.5$ were considered malnourished. This was done according to Asian classification. After ATT was started, the patients were monitored for the development of symptoms of DILI. Liver function tests were done at the baseline, on the third day and at discharge in all the patients. The frequency of LFT was increased depending on the risk factors and development of symptoms. This was done at the treating physician's discretion. DILI was defined as an increase in serum aminotransferase level $>5$ times the upper limit of normal (ULN) in asymptomatic individuals and $>3$ times the ULN in symptomatic patients. In patients diagnosed with DILI, ATT medications were withheld and were reintroduced sequentially once liver function tests were normalized. The offending drug was identified and replaced with an alternative drug as per the judgement of treating physician. Those patients who chose to continue treatment at our hospital were followed up until the end of the study period.

Data analysis: Chi-square tests and conditional multiple logistic analysis was performed to identify risk factors associated with DILI.

\section{Results and Discussion}

In this study, a total of 319 patients were enrolled. A total of 188 of these were diagnosed with pulmonary TB (PTB) (58.9\%), and the remaining 131 were diagnosed with extrapulmonary TB (EPTB) (41.06\%). Majority of the subjects of the total cohort were male (male, 209; female, $110)$ and belonged to the age group of $40-49$ years. The mean age was $46.3 \pm 16.62$ years. PTB was prevalent in the age group 40-49, whereas EPTB was prevalent in the 20-29 age group. It was found that $35.7 \%$ of these subjects were under-nourished. The mean BMI was 20.3 \pm 4.01 . The proportion of individuals with low BMI was significantly higher in PTB than in EPTB (45\% vs. $22 \%$, $p<0.001)$.

Out of the 319 subjects, 181 (56.7\%) were transferred out to their respective directly observed treatment shortcourse chemotherapy (DOTS) centre while the remaining $138(43.2 \%)$ chose to follow up at our hospital.

Of the 138 patients who were managed at our hospital, a total of $45(32.6 \%)$ of these patients were cured, 65 (47.1\%) completed treatment, 19 (13.8\%) were still on ATT, and a total of nine $(6.5 \%)$ patients died during treatment. A total of 14 patients (10\%) developed DILI. All patients who developed DILI were initially started on four-drug ATT. The median time to onset of DILI was found to be ten days. The primary drug attributed to causing this liver injury was found to be pyrazinamide, seen in about 8 of the 14 cases. This was followed by rifampicin, seen in 2 cases. Others were undetermined.

A total of $20 \%$ of the patients who were malnourished developed DILI while only $5.4 \%$ of the patients with a BMI of $>18.5 \mathrm{~kg} / \mathrm{m}^{2}$ developed DILI. BMI was found to have a significant association with DILI. EPTB and high baseline transaminase levels were also found to have a significant association with DILI (Table 1). However, other attributed risk factors such as pre-existing liver disease, low serum albumin levels, female sex and human immunodeficiency virus (HIV) coinfection were not associated with increased risk for DILI. Low serum albumin had increased odds ratio but wasn't statistically significant.

DILI is a possible adverse outcome of the consumption of ATT. DILI is one of the leading causes of treatment

Table 1. Chi-square test and conditional multivariate logistic regression analysis to determine factors associated with DILI

\begin{tabular}{|c|c|c|c|c|}
\hline Items & DILI, No. (\%) & non-DILI, No. (\%) & $p$-value & OR $(95 \% \mathrm{CI})$ \\
\hline Sex & & & 0.827 & $1.137(0.702-2.088)$ \\
\hline Male & $9(10.6)$ & $76(89.4)$ & & \\
\hline Female & $5(9.4)$ & $48(90.6)$ & & \\
\hline Type & & & 0.034 & $0.15(0.35-0.64)$ \\
\hline РTB & $4(6)$ & $63(94)$ & & \\
\hline ЕРТВ & $10(14.1)$ & $61(85.9)$ & & \\
\hline BMI & & & 0.025 & - \\
\hline$<18.5$ & $9(20)$ & $36(80)$ & & \\
\hline $18.5-22.9$ & $2(3.8)$ & $50(96.2)$ & & \\
\hline$\geq 23$ & $3(7.3)$ & $38(92.7)$ & & \\
\hline Albumin & & & 0.207 & $2.024(0.38-6.22)$ \\
\hline$<3.5$ & $7(14.6)$ & $41(85.4)$ & & \\
\hline$\geq 3.5$ & $7(7.8)$ & $83(92.2)$ & & \\
\hline HIV & $2(20)$ & $8(80)$ & 0.284 & 0.414 \\
\hline $\mathrm{HBV}$ & $0(0)$ & $2(100)$ & 0.632 & 1.016 \\
\hline $\mathrm{HCV}$ & $0(0)$ & $2(100)$ & 0.632 & 1.016 \\
\hline CLD & $0(0)$ & $5(25)$ & 0.491 & 1.051 \\
\hline Baseline ALT & $56 \pm 95.9$ & $27.7 \pm 28.3$ & 0.056 & $0.99(0.98-1.0)$ \\
\hline
\end{tabular}

*DILI, drug-induced liver injury; OR, odds ratio; CI, confidence interval; BMI, body mass index; HIV, human immunodeficiency virus; HBV, hepatitis B virus; $\mathrm{HCV}$, hepatitis C virus; CLD, chronic liver disease; ALT, alanine transferase. 
Table 2. Comparison of DILI and attributed risk factors among various studies

\begin{tabular}{|c|c|c|c|c|c|c|}
\hline Studies & Location of study & $\begin{array}{c}\text { No. of patients } \\
\text { enrolled }(\mathrm{N})\end{array}$ & $\begin{array}{l}\text { Duration of } \\
\text { study (years) }\end{array}$ & $\begin{array}{l}\text { Incidence } \\
\text { of DILI (\%) }\end{array}$ & Risk factors attributed & $\begin{array}{l}\text { Median time } \\
\text { of onset (days) }\end{array}$ \\
\hline Current study & Manipal, India & 134 & 2 & $14(10)$ & Low BMI, EPTB, high baseline ALT & 10 \\
\hline $\begin{array}{l}\text { Saha et al. } \\
(2008-2012)(8)\end{array}$ & Vellore, India & 252 & 5 & $26(9.5)$ & Nil & \\
\hline $\begin{array}{l}\text { Latief et al. } \\
(2012-2015)(7)\end{array}$ & $\begin{array}{l}\text { Jammu \& Kashmir, } \\
\text { India }\end{array}$ & 200 & 3 & $16(8.4)$ & Female gender, EPTB & $<14$ \\
\hline $\begin{array}{l}\text { Naidoo et al. } \\
(2009-2012)(11)\end{array}$ & South Africa & 94 & 3 & & Age $>35$ years, Low Albumin & 31 \\
\hline $\begin{array}{l}\text { Abbara et al. } \\
(2010-2014)(9)\end{array}$ & England & 1,529 & 4 & $105(6.9)$ & $\begin{array}{l}\text { Low BMI, HIV-TB coinfection, } \\
\text { Alcohol, High baseline ALP }\end{array}$ & 12.5 \\
\hline $\begin{array}{l}\text { Tweed et al. } \\
(2018)(10)\end{array}$ & London & 1,927 & & $58(3)$ & Older age, Asian ethnicity & \\
\hline
\end{tabular}

*DILI, drug-induced liver injury; BMI, body mass index; HIV, human immunodeficiency virus; ALT, alanine transferase; ALP, alkaline phosphatase; TB, tuberculosis; EPTB, extrapulmonary tuberculosis.

interruption in patients on ATT. The risk factors of DILI in previously published studies have been compiled in Table 2. According to Saukkonen et al., the incidence of DILI attributable to ATT is $5-33 \%$ (6). A total of $10 \%$ of the patients developed DILI in our study. Similar results were observed in studies conducted in India by Latief et al. (2012-2015), and Saha et al. (2008-2012) wherein the incidence rates for DILI were found to be $8.4 \%$ and $9.5 \%$ respectively $(7,8)$. The median time of onset of DILI was found to be within ten days of starting the treatment with ATT. This correlates well with similar studies done by Abbara et al. wherein it was found to begin within 12.5 days of treatment initiation (9). Other studies from India have also reported DILI onset to be common in the first two weeks of starting ATT $(7,8)$. Therefore, patients with risk factor, who are started on ATT, should be followed for the first two weeks of treatment with LFTs. This will help in early identification of DILI.

Three of the five first-line ATT drugs have been known to cause hepatotoxicity, i.e. isoniazid, pyrazinamide, and rifampicin. Pyrazinamide has been documented as being the most hepatotoxic, according to Saukkonen et al. (6). The drug that most commonly attributed to the development of DILI in our study was also pyrazinamide (in 8 of the 14 patients).

Malnutrition plays a two-fold role in increasing the morbidity and mortality rates of people suffering from TB. Nutritional depletion has a major impact on immune function, and loss of CMI is not an advantageous factor in an individual fighting an invasive mycobacterial infection. Primary malnutrition is known to not only increase the frequency of occurrence but also to exacerbate clinical manifestations of TB. In addition to ATT, these patients require an adequate supply of nutrition during the treatment/ recovery phase. In our study, patients with low BMI were significantly at a higher risk of developing DILI. Malnutrition increases the risk of developing DILI due to altered drug metabolism pathways (9).

Increasing age has been reported as a risk factor of DILI in several studies. This is because of the agerelated decline in liver function. A study was done by Naidoo et al., found a higher incidence of DILI in patients above the age of 35 years (10). Similar results were observed by Tweed et al., who found that DILI was more common in the elderly. In our study, the age difference between the two groups was statistically insignificant ( $p$-value 0.261 ). No association with gender and DILI was found in our study, though certain studies done in North India have found an association with female gender and DILI (7). Interestingly, the same study found an association with EPTB and DILI, as was the case in our study. This may be due to subclinical involvement of the liver as a part of EPTB. However, the exact reason for the same remains unclear. Though HIV-TB coinfection and association with DILI have been reported previously, this association was not found in our current study. Similar results have also been observed in a study done by Tweed $e t$ al. in England (11). In that study, an increased number of HIV-TB coinfection patients showed elevated liver enzymes, but that result was not statistically significant (7). A study was done in South India, which also failed to find such an association (7). Sharma et al. reported low serum albumin as an independent risk factor for the development of DILI (12). Low serum albumin level as a risk factor for DILI was reported in studies done in South Africa as well (10). However, in our study and other studies done in India, low albumin level was not found to be a risk factor for the development of DILI $(7,8)$.

In conclusion, malnutrition, presence of EPTB and pre-existing increased liver enzymes are an important 
risk factor for TB-DILI. Further studies are needed to evaluate whether nutritional interventions, along with ATT, will help in mitigating this risk.

\section{Acknowledgements}

Authors express their sincere gratitude to all the voluntary participants of this study.

\section{References}

1. Global Tuberculosis Report 2018. Geneva: World Health Organization; 2018. Available from: https://apps.who.int/ medicinedocs/en/m/abstract/Js23553en/ (accessed Mar 12, 2020)

2. Martin SJ, Sabina EP. Malnutrition and associated disorders in tuberculosis and its therapy. J Diet Suppl. 2019; 16:602-610.

3. Bhargava A. Undernutrition, nutritionally acquired immunodeficiency, and tuberculosis control. BMJ. 2016; 355:i5407.

4. Devarbhavi H. Antituberculous drug-induced liver injury: current perspective. Trop Gastroenterol Off J Dig Dis Found. 2011; 32:167-174.

5. Tostmann A, Boeree MJ, Aarnoutse RE, de Lange WCM, van der Ven AJAM, Dekhuijzen R. Antituberculosis drug-induced hepatotoxicity: concise up-to-date review. J Gastroenterol Hepatol. 2008; 23:192-202.

6. Saukkonen JJ, Cohn DL, Jasmer RM, Schenker S, Jereb JA, Nolan CM, Peloquin CA, Gordin FM, Nunes D, Strader DB, Bernardo J. An official ATS statement: hepatotoxicity of antituberculosis therapy. Am J Respir Crit Care Med. 2006; 174:935-952.

7. Latief M, Dar WR, Sofi N, Dar IA, Kasana B, Hussain M, Arshad F, Shah BA, Koul PA. Novel risk factors and early detection of anti tubercular treatment induced liver injury - Looking beyond American Thoracic Society Guidelines. Indian J Tuberc. 2017; 64:26-32.

8. Saha A, Shanthi FX M, Winston A B, Das S, Kumar A, Michael JS, Balamugesh T. Prevalence of hepatotoxicity from antituberculosis therapy: a five-year experience from South India. J Prim Care Community Health. 2016; 7:171-174

9. Abbara A, Chitty S, Roe JK, Ghani R, Collin SM, Ritchie A, Kon OM, Dzvova J, Davidson H, Edwards TE, Hateley C. Drug-induced liver injury from antituberculous treatment: a retrospective study from a large TB centre in the UK. BMC Infect Dis. 2017; 17:231.

10. Naidoo S, Evans D, Jong E, Mellet K, Berhanu R. Outcomes of TB/HIV co-infected patients presenting with antituberculosis drug-induced liver injury. S Afr Med J. 2015; 105:393-396.

11. Tweed CD, Crook AM, Amukoye EI, Dawson R, Diacon AH, Hanekom M, McHugh TD, Mendel CM, Meredith SK, Murphy ME, Murthy SE. Toxicity associated with tuberculosis chemotherapy in the REMoxTB study. BMC Infect Dis. 2018; 18:317.

12. Sharma SK, Balamurugan A, Saha PK, Pandey RM, Mehra NK. Evaluation of clinical and immunogenetic risk factors for the development of hepatotoxicity during antituberculosis treatment. Am J Respir Crit Care Med. 2002; 166:916-919.

Received May 13, 2020; Revised June 21, 2020; Accepted June 26, 2020.

*Address correspondence to:

Kavitha Saravu, Department of Infectious diseases, Kasturba Medical College, Manipal- 576104, Karnataka, India.

E-mail-kavithasaravu@gmail.com 